\title{
Essai de mise en évidence d'une évolution récente du pH et de la teneur en cations « basiques » de quelques sols forestiers des Vosges (nord-est de la France)
}

\author{
Y Lefèvre \\ Unité écophysiologie forestière, centre de recherches Inra de Nancy, \\ 54280 Champenoux, France
}

(Reçu le 26 mars 1996 ; accepté le 26 novembre 1996)

\begin{abstract}
Summary - An assessment of the recent trend of $\mathrm{pH}$ and base cation content in some forest soils in the Vosges Mountains (north-east France). In two series of sites in the Vosges massif, soils were sampled after a 20 year interval. The comparison of the base cation content of the organomineral horizon Al showed high exchangeable calcium and magnesium losses, which are only statistically significant in the 'cartographie' series. In these soils the mean saturation level of the AI horizons decreased from 10.8 to $7.3(32 \%)$; however, pH was not affected. In podzolic soils in the 'Hospices de Nancy' series, an increase in organic carbon content, and a subsequent increase in the CEC, produce uncertainties with respect to data interpretation. In brown soils, however, a true decrease in the saturation rate seems to have occurred during this short period.
\end{abstract}

acidification / historical comparison / soil change

Résumé - Dans deux séries de stations du massif des Vosges, les sols ont été échantillonnés à 20 ans d’intervalle. La comparaison, qui porte essentiellement sur la teneur en cations « basiques » des horizons organominéraux $A_{1}$, met en évidence d’importantes pertes en calcium et en magnésium échangeables statistiquement significatives dans la seule série « Cartographie ». Dans cette série, le taux de saturation dans les horizons $\mathrm{A}$, passe en moyenne de 10,8 à 7,3, soit une baisse de $32 \%$; en revanche, le $\mathrm{pH}$ n'est pas affecté. Un accroissement de la teneur en carbone organique et, par conséquent, une augmentation de la CEC incite à la prudence dans l'interprétation du phénomène dans les sols podzoliques de la série « Hospices de Nancy ». En revanche, dans les sols bruns, une aggravation de l'état de désaturation du complexe absorbant sur une période courte semble bien réelle.

acidification / évolution du sol / étude diachronique

Tél : (33) 0383394083 ; fax : (33) 0383394069 


\section{INTRODUCTION}

Parmi les causes du dépérissement forestier, une acidification accrue des sols par les apports atmosphériques est souvent retenue, au moins comme facteur aggravant (Bonneau, 1989). Dans divers pays européens (Allemagne, Suède, Grande-Bretagne), des études de type diachronique ont mis en évidence une acidification rapide des sols, qui s'exprime tant par une baisse du pH (Tamm et Hallbäcken, 1988) que par une désaturation du complexe d'échange (Johnson et al, 1991).

Il a paru opportun de vérifier si ce phénomène était observable en France, en particulier dans le massif des Vosges où un dépérissement des peuplements forestiers a été largement observé depuis les années 1980. Cette étude se limite intentionnellement à l'observation d'une évolution ; d'autres recherches, fondées sur les méthodes de bilan, traitent de l'aspect explicatif.

\section{MATÉRIEL ET MÉTHODES}

\section{Cadre géographique}

La condition nécessaire pour réaliser ce type d'étude est de disposer de données recueillies sur des sites connus avec une grande précision. Un premier choix (Landmann, 1989) a été d'échantillonner de nouveau les humus des parcelles 10 et 11 de la forêt des Hospices de Nancy, située sur le versant lorrain du massif du Louchbach (Vosges). Cette zone d'environ 20 ha, climatiquement homogène (faible variation altitudinale, exposition peu variable) et reposant sur une seule roche mère (granite du Valtin), présente une variabilité importante des types d'humus et de sol (Chichery, 1970). Parmi ceux initialement étudiés, 12 points repérés selon un maillage systématique ont été retenus. Dans cette série appelée " Hospices de Nancy », les sols varient du sol brun acide au podzol ; lc peuplement est une sapinière traitée en futaie régulière, qui avait 100 ans d'âge moyen en 1970 .

L'extension de ces observations, tout en restant dans le contexte du massif vosgien, a nécessité la recherche d’autres sites. Les données recueillies lors des travaux de cartographie (année 1970) de la feuille au 1/100000 de Saint-Dié (Vosges) ont donc été utilisées. Après vérification sur le terrain de la précision de l'emplacement et de l'absence de perturbation majeure dans l'état du site (mise en régénération, travaux divers), 11 stations réparties dans les basses et moyennes Vosges gréseuses et une station en bordure des hautes Vosges granitiques ont été sélectionnées. Toutes ont conservé une couverture forestière complète ayant subi, outre le vieillissement naturel, un régime d'intervention sylvicole normal (passage en éclaircie). Les caractéristiques des sites de cette série appelée «cartographie » sont décrits dans le tableau I.

\section{Prélèvements}

Dans la série Hospices de Nancy, seul l'horizon organominéral $A_{1}$ a été échantillonné en 1970. Un échantillon moyen avait été constitué du mélange de quatre prélèvements différents réalisés à proximité immédiate des points positionnés sur le plan de la parcelle. En 1986, la même technique a été appliquée.

Chaque placette de la série Cartographie, a été échantillonnée en 1970 et en 1992. En 1970, un échantillon moyen de l'horizon organominéral $A_{1}$, constitué du mélange de trois ou quatre prélèvements, a été analysé. En 1992, l'horizon $A_{1}$ a été prélevé sur la même épaisseur que celle définie en 1970 en cinq endroits répartis dans un rayon d'une dizaine de mètres autour du prélèvement de 1970. Ces cinq échantillons ont été analysés séparément pour apprécier la variabilité spatiale. En 1970, le prélèvement du premier horizon minéral $\mathrm{A}_{2}$ ou $(\mathrm{B})$ avait été réalisé sur les différentes paroîs de la même fosse. En 1992, ce prélèvement a été réalisé sur trois fosses entre 20 et $30 \mathrm{~cm}$ de profondeur (en respectant les limites d'horizon pédologique), puis mélangé.

\section{Analyses}

Les déterminations chimiques ont été réalisées, aux deux dates, dans le même laboratoire (Inra d'Arras) et selon les mêmes protocoles. Les analyses portent sur la terre fine $(<2 \mathrm{~mm})$ sèche à l'air.

Le carbone organique est dosé selon la méthode Anne et l'azote total selon la méthode Kjeldhal. La détermination de la capacité 
Tableau I. Principales caractéristiques des sites de la série Cartographie.

\begin{tabular}{|c|c|c|c|c|c|}
\hline Dénomination & $N^{o}$ code & Nature du peuplement & Altitude (m) & Roche mère & Type de sol \\
\hline Petit Paris & 21 & Futaie de hêtre & 370 & $\begin{array}{l}\text { Colluvium grès } \\
\text { vosgien } \\
\text { et intermédiaire }\end{array}$ & Brun acide \\
\hline Forêt de Bru & 81 & Futaie de hêtre et de chêne & 400 & $\begin{array}{l}\text { Grès à Voltzia } \\
\text { faciès sableux }\end{array}$ & Brun ocreux \\
\hline Forêt d'Anglemont & 10 & Vieille futaie de hêtre & 375 & $\begin{array}{l}\text { Grès à Voltzia } \\
\text { faciès argileux }\end{array}$ & Brun acide \\
\hline Rott à Moncel & 14 & Futaie de hêtre & 375 & Grès intermédiaire & Brun acide \\
\hline Grands fourchons & 171 & Futaie de chêne et & 430 & Grès intermédiaire & Brun ocreux \\
\hline Col de Trace & 18 & $\begin{array}{l}\text { Futaie de sapin } \\
\text { et régénération }\end{array}$ & 440 & Grès vosgien & Ocre podzolique \\
\hline Grands fins & 20 & Futaie de pin sylvestre & 310 & Grès & Podzo \\
\hline Bois de l'Aunot & 22 & Jeune futaie de hêtre & 360 & Grès intermédiaire & Brun acide \\
\hline Ormont & 322 & Futaie de pin sylvestre & 640 & grès vosgien & Podzol \\
\hline Haut Jac & 401 & Futaie de hêtre et de sapin & 600 & Grès intermédiaire & Brun acide \\
\hline Biffontaine & 403 & Futaie de pin sylvestre & 520 & Grès vosgien & Podzol \\
\hline Plainfaing & 404 & Futaie de sapin & 790 & Granite du Valtin & Brun ocreux \\
\hline
\end{tabular}

d'échange cationique (CEC ou T) est obtenue par la méthode de Metson qui utilise l'acétate d'ammonium $\mathrm{IN}$ à $\mathrm{pH} 7$ comme solution d'échange. Les bases échangeables, $\mathrm{Ca}^{2+}, \mathrm{Mg}^{2+}$ et $\mathrm{K}^{+}$, sont dosées en milieu acétate. L'aluminium échangeable $\left(\mathrm{Al}^{3+)}\right.$ est dosé selon la méthode Jackson (échange au $\mathrm{KCl} / \mathrm{N}$ ). Le $\mathrm{pH}$ eau est mesuré sur une suspension avec un rapport pondéral sol/solution $=1 / 2.5$.

On notera que, bien que les mêmes méthodes analytiques aient été appliquées, les techniques de dosage se sont perfectionnées pour certains éléments, ce qui peut induire des différences de précision mais non d'ordre de grandeur.

\section{RESULTATS}

\section{Série Hospices de Nancy}

La comparaison entre les teneurs moyennes de 1970) et de 1986 montre une augmentation dans une même proportion du carbone et de l'azote et, en conséquence, une stabilité du rapport $\mathrm{C} / \mathrm{N}$ (tableau II). Parallèlement, l'augmentation de la CEC est nettement corrélée à celle du taux de carbone $\left(\mathrm{R}^{2}\right.$ de 0,57$)$. Ainsi la contribution de la matière

Tableau II. Caractérisation physicochimique des horizons organominéraux $\left(\mathrm{A}_{1}\right)$ de la série des Hospices de Nancy.

\begin{tabular}{|c|c|c|c|c|c|c|c|c|c|c|}
\hline & $C$ & $N$ & $C / N$ & $\mathrm{Ca}^{2+}$ & $M g^{2+}$ & $K^{+}$ & $C E C$ & $S / T$ & $\mathrm{Al}^{3+}$ & $\begin{array}{l}S / T \text { all pH } \\
\text { dlu soll }\end{array}$ \\
\hline & $\%$ & $\%$ & & meq & meg & mequ & meq & $\%$ & meq & $\%$ \\
\hline 1970 & 6,73 & 0,38 & 17,8 & 0,57 & 0,2 & 0 & 17 & 6.3 & 6,12 & 15,9 \\
\hline 1986 & 9.13 & 0.52 & 17.5 & 0,49 & 0,21 & 0.28 & 22.9 & 4.8 & 7.06 & 12,4 \\
\hline$\Delta$ & 2,4 & 0,14 & -0.3 & $-0,08$ & 0,01 & 0.0 .3 & 5,4 & $-1,5$ & 0.94 & -3.5 \\
\hline en $\%$ & 35,7 & 36,8 & -1.7 & $-14,0$ & 5.0 & 12.0 & 30.9 & $-23,8$ & 15,4 & $-22,0$ \\
\hline $\mathrm{P}>\left.|7|\right|^{b}$ & $0,009 \div$ & $0,003: \leqslant$ & $0,875 \mathrm{~ns}$ & $0,346 \mathrm{~ns}$ & $0.688 \mathrm{~ns}$ & $0,040 *$ & $0.009 *$ & $0.009: k: k$ & $0,099 *$ & $0,209 \mathrm{~ns}$ \\
\hline
\end{tabular}

"S/T calculé au $\mathrm{pH}$ du sol : $\sum$ bases échangeables/ $\Sigma$ bases échangeables + Al échangeable ; probabilité associée au test du signe et rang : ns: non significatif, * significatit au seuil de 0,05 , ** signilicaltil au seuil de 0,01 . 


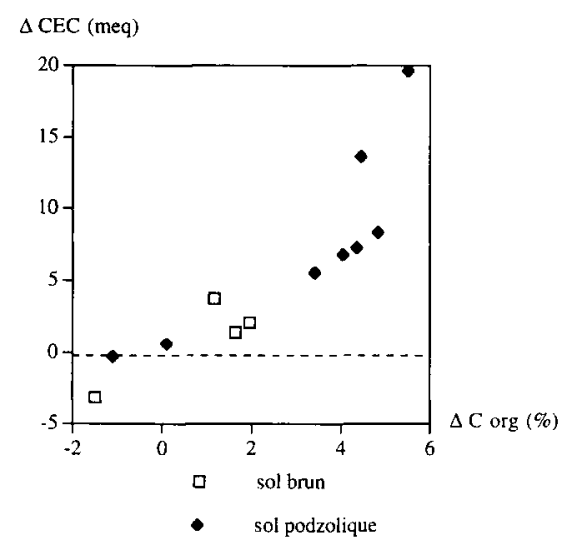

Fig 1. Variation de la capacité d'échange cationique ( $\triangle$ CEC) entre 1970 et 1986 en fonction de la variation de la teneur en carbone organique $(\Delta C)$ dans les horizons $A_{1}$ de la série des Hospices de Nancy.

organique à la CEC totale a augmenté, ce qui explique en grande partie la baisse significative du taux de saturation (S/T). Néanmoins, alors que la teneur moyenne en $\mathrm{Mg}$ est stable et que celle en $\mathrm{K}$ augmente légèrement, la baisse sensible $(-14 \%)$ mais non significative de la teneur moyenne en Ca contribue probablement à l'explication de la désaturation du complexe absorbant. En fait, cette désaturation est attestée plus clairement par l'augmentation significative de la teneur en $\mathrm{Al}$ échangeable.

Ces observations sur des moyennes masquent une forte variabilité. L'amplitude des variations des teneurs en carbone est importante (fig 1), et plus particulièrement dans les sols podzolisés. Cependant, une faible imprécision dans l'épaisseur prélevée d'un horizon $A_{1}$ peut entraîner d'importants écarts sur la teneur en matière organique, en présence d'horizons holorganiques juxtaposés. En revanche, dans les sols brunifiés, ce risque d'erreur est bien moindre. Par ailleurs, il est probable que les phénomènes d'évolution s'expriment plus nettement dans les sols initialement les moins désaturés.
Dans cette série, deux groupes peuvent être déterminés, les sols brunifiés qui avaient un $\mathrm{S} / \mathrm{T}$ moyen de 11 à $\mathrm{pH} 7$ en 1970, d'une part, et les sols podzolizés, d'autre part, qui avaient un S/T moyen de 4 à $\mathrm{pH} 7$ cette même année. Dans les sols bruns, la baisse moyenne du taux de saturation est d'environ $30 \%$ et celle du calcium échangeable de $50 \%$ (figs 2 et 3 ),

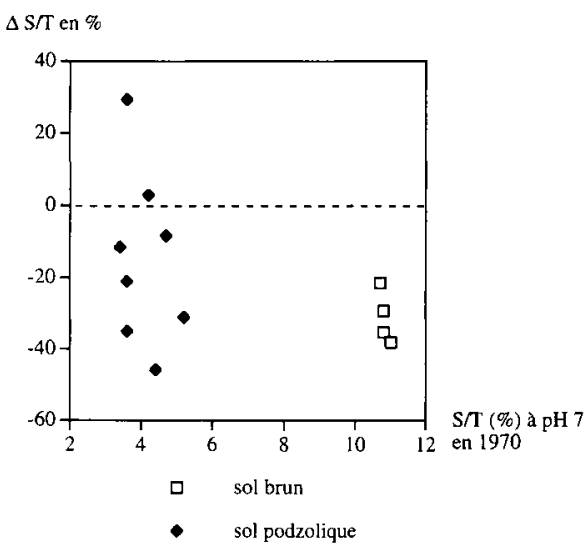

Fig 2. Variation du taux de saturation $(\Delta \mathrm{S} / \mathrm{T})$ à $\mathrm{pH} 7$ entre 1970 et 1986 en fonction de sa valeur initiale dans les horizons $\mathrm{A}_{1}$ de la série des Hospices de Nancy.

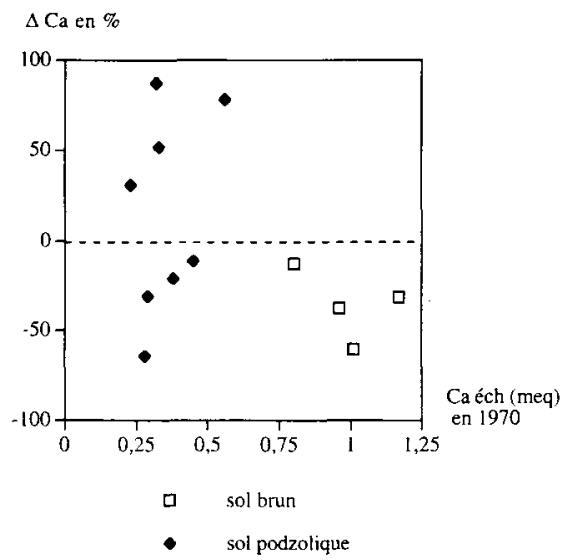

Fig 3. Variation de la teneur en calcium échangeable $\left(\Delta \mathrm{Ca}^{2+}\right)$ entre 1970 et 1986 en fonction de sa valeur initiale dans les horizons $\mathrm{A}_{1}$ de la série des Hospices de Nancy. 
avec des probabilités associées à un test $t$ de respectivement 0,004 et 0,041 pour $\mathrm{S} / \mathrm{T}$ et $\mathrm{Ca}$, or c'est dans ce groupe que les variations de la teneur en $\mathrm{C}$ et par conséquent de la CEC sont les plus faibles. La désaturation observée est donc directement liée à la perte en $\mathrm{Ca}$. Parallèlement, on remarque une augmentation sensible de la concentration en Al échangeable, ce qui explique que le phénomène est encore plus net sur l'évolution du taux de saturation au $\mathrm{pH}$ du sol $(-50 \%)$ (fig 4$)$.

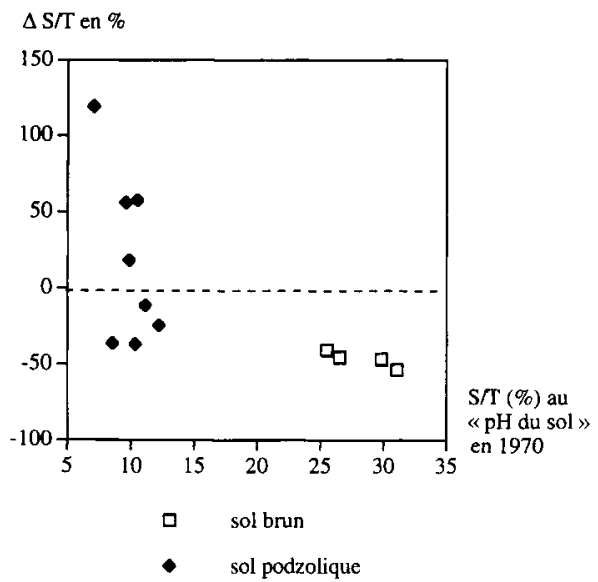

Fig 4. Variation du taux de saturation au « $\mathrm{pH}$ du sol $\gg(\Delta S / T)$ entre 1970 et 1986 en fonction de sa valeur initiale dans les horizons $A_{1}$ de la série des Hospices de Nancy.

\section{Série Cartographie}

\section{Horizons organominéraux $A_{I}$}

En 22 ans, les teneurs moyennes en carbone et en azote et la valeur du rapport $\mathrm{C} / \mathrm{N}$ sont restées globalement stables (tableau III). Dans le détail, le rapport $\mathrm{C} / \mathrm{N}$ a eu tendance à baisser (fig 5) dans les placettes où sa valeur initiale était élevée (sols podzoliques). Toutefois, en raison du faible effectif de ce groupe (quatre placettes) et du comporte-

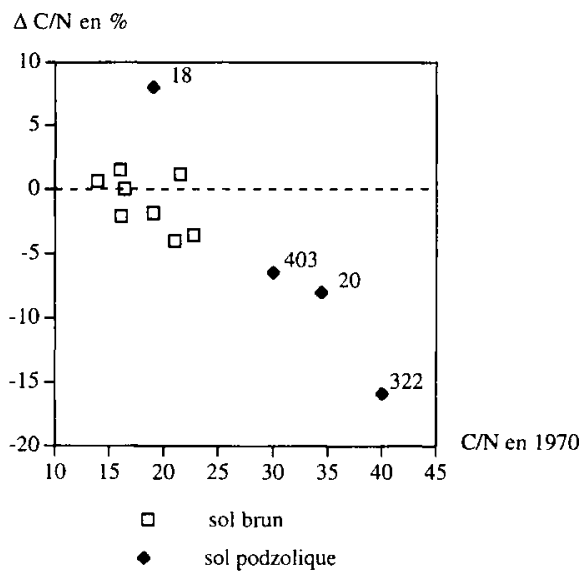

Fig 5. Variation du rapport carbone organique sur azote total $(\Delta \mathrm{C} / \mathrm{N})$ entre 1970 et 1992 en fonction de sa valeur initiale dans les horizons $A$, de la série Cartographie.

Tableau III. Caractérisation physicochimique des horizons organominéraux $\left(\mathrm{A}_{1}\right)$ de la série Cartographie.

\begin{tabular}{lccccccccc}
\hline & $p H$ & $\begin{array}{c}C \\
\%\end{array}$ & $\begin{array}{c}N \\
\%\end{array}$ & $C / N$ & $\begin{array}{c}C a^{2+} \\
m e q\end{array}$ & $\begin{array}{c}M g^{2+} \\
m e q\end{array}$ & $\begin{array}{c}K^{+} \\
m e q\end{array}$ & $\begin{array}{c}C E C \\
m e q\end{array}$ & $\begin{array}{c}S / T \\
\%\end{array}$ \\
\hline 1970 & 4,1 & 5,45 & 0,27 & 22,5 & 0,77 & 0,28 & 0,28 & 13,98 & 10,8 \\
1992 & 4,1 & 5,93 & 0,30 & 19,9 & 0,42 & 0,15 & 0,24 & 12,75 & 7,3 \\
$\Delta$ & & 0,48 & 0,03 & $-2,5$ & $-0,35$ & $-0,13$ & $-0,04$ & $-1,23$ & $-3,5$ \\
en $\%$ & 8,8 & 11,0 & $-11,5$ & -45.5 & $-46,4$ & $-14,3$ & $-8,8$ & $-32,4$ \\
P>|z|a & & $0,937 \mathrm{~ns}$ & $0,272 \mathrm{~ns}$ & $0,157 \mathrm{~ns}$ & $0,003^{*} *$ & $0,009^{* *}$ & $0,999 \mathrm{~ns}$ & $0,530 \mathrm{~ns}$ & $0,029^{*}$
\end{tabular}

¿Voir tableau II. 

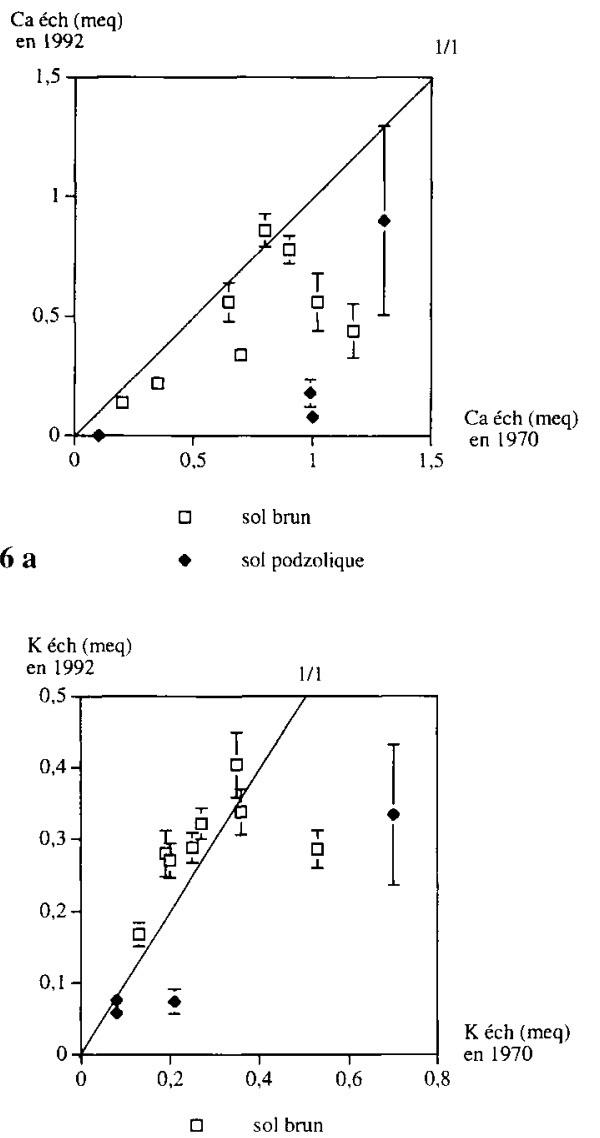

$6 \mathrm{c}$

- sol podzolique

ment particulier de la placette 18 liée à une évolution du peuplement (fermeture du couvert sous une régénération surabondante de sapin pectiné), cet effet n'est pas significatif.

Aucune évolution sur le $\mathrm{pH}$ n'est décelable dans cette série de mesures.

La teneur moyenne en calcium échangeable diminue significativement et dans une fortc proportion ( $45 \%$ ). Le phénomène est général, excepté pour une placette. La diminution du magnésium échangeable est du même ordre de grandeur (46\%). En revanche, l'évolution de la teneur en potassium échangeable n'est sensible que dans quelques sites (figs $6 a, b, c)$. La capacité

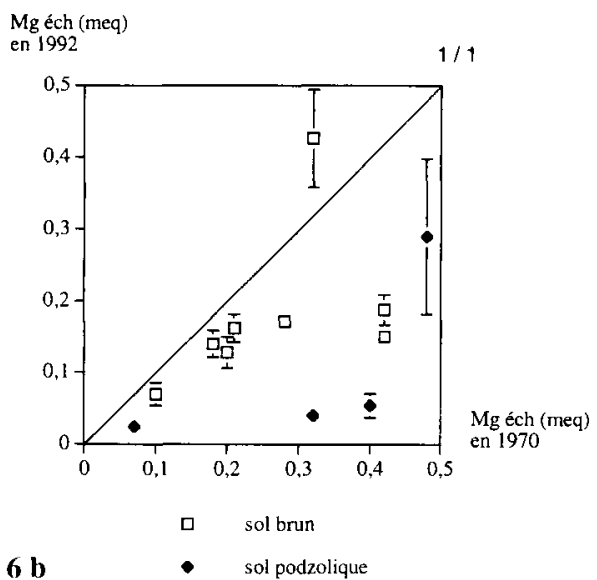

Fig 6 a, b, c. Évolution des teneurs moyennes en bases échangeables $\left(\mathrm{Ca}^{2+}, \mathrm{Mg}^{2+}, \mathrm{K}^{+}\right)$entre 1970 et 1992 dans les horizons $A_{1}$ de la série Cartographie (moyenne \pm erreur standard de la valeur de 1992).

d'échange cationique est stable dans la plupart des placettes. Le taux de saturation diminue significativement et passe de 10,8 en 1970 à 7,3 en 1992, soit une perte de $32 \%$. La diminution est d'autant plus forte que la valeur initiale est élevée (fig 7).

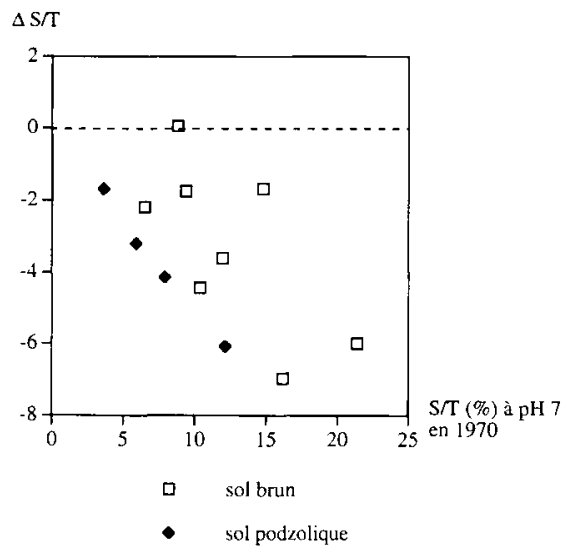

Fig 7. Variation du taux de saturation $(\Delta S / T)$ entre 1970 et 1992 en fonction de sa valeur initiale dans les horizons $\mathrm{A}_{1}$ de la série Cartographie. 
Tableau IV. Caractérisation physicochimique des horizons minéraux $(20-30 \mathrm{~cm})$ de la série Cartographie.

\begin{tabular}{lccccccccc}
\hline & $\mathrm{pH}$ & $\begin{array}{c}\mathrm{C} \\
\%\end{array}$ & $\begin{array}{c}\mathrm{N} \\
\%\end{array}$ & $\mathrm{C} / \mathrm{N}$ & $\begin{array}{c}\mathrm{Ca}^{2+} \\
\mathrm{meq}\end{array}$ & $\begin{array}{c}\mathrm{Mg}^{2+} \\
\mathrm{meq}\end{array}$ & $\begin{array}{c}\mathrm{K}^{+} \\
\mathrm{meq}\end{array}$ & $\begin{array}{c}\mathrm{CEC} \\
\mathrm{meq}\end{array}$ & $\begin{array}{c}\mathrm{S} / \mathrm{T} \\
\%\end{array}$ \\
\hline 1970 & 4,4 & 1,09 & 0,05 & 22,1 & 0,15 & 0,08 & 0,07 & 5,0 & 7,5 \\
1992 & 4,5 & 1,33 & 0,07 & 20,6 & 0,03 & 0,02 & 0,06 & 5,18 & 2,7 \\
$\Delta$ & 0,1 & 0,24 & 0,02 & $-1,5$ & $-0,12$ & $-0,06$ & $-0,01$ & 0,18 & $-4,8$ \\
en \% & & 22,0 & 40,0 & $-6,8$ & $-80,0$ & $-75,0$ & $-14,3$ & 3,6 & $-64,0$ \\
$\mathrm{P}>|\mathrm{z}|^{\text {a }}$ & & $0,013^{*}$ & $0,010^{*}$ & $0,480 \mathrm{~ns}$ & $0,213 \mathrm{~ns}$ & $0,003 * *$ & $0,392 \mathrm{~ns}$ & $0,724 \mathrm{~ns}$ & $0,049 *$ \\
\hline
\end{tabular}

a Voir tableau II.

\section{Horizons minéraux $(20-30 \mathrm{~cm})$}

Dans les horizons minéraux, la teneur en azote est en nette augmentation (40\%) (tableau IV). L'augmentation de la teneur en carbone, bien que moins prononcée (fig 8), limite en conséquence la fluctuation du rapport $\mathrm{C} / \mathrm{N}$ qui n'est pas significative.

Les teneurs moyennes en calcium échangeable et en magnésium échangeable baissent fortement alors que celle en potassium

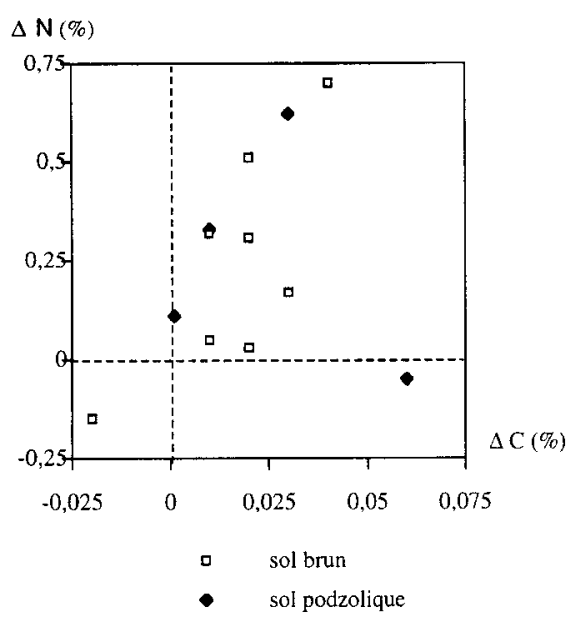

Fig 8. Variation de la teneur en carbone organique ( $\triangle \mathrm{C}$ org) et de la teneur en azote total $(\Delta \mathrm{N})$ entre 1970 et 1992 dans le premier horizon minéral de la série Cartographie. échangeable est relativement stable. Ces observations sur des moyennes masquent une grande dispersion. La différence en $\mathrm{Ca}$ (non significative sur la moyenne) n'apparaît comme nette que dans les sols podzoliques (fig 9); en revanche, la baisse en $\mathrm{Mg}$ (significative sur la moyenne) s'observe dans les deux groupes de sol. Le taux de saturation passe en moyenne de 7,5 à 2,7, mais sa variation est d'amplitude très différente selon les sites (fig 10), ce qui en rend difficile l'interprétation.

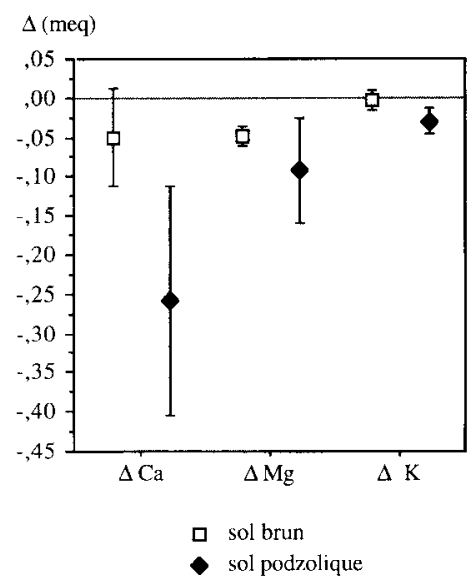

Fig 9. Variation des teneurs en bases échangeables $\left(\Delta \mathrm{Ca}^{2+}, \Delta \mathrm{Mg}^{2+}, \Delta \mathrm{K}^{+}\right)$entre 1970 et 1992 dans le premier horizon minéral de la série Cartographie (moyenne \pm erreur standard). 


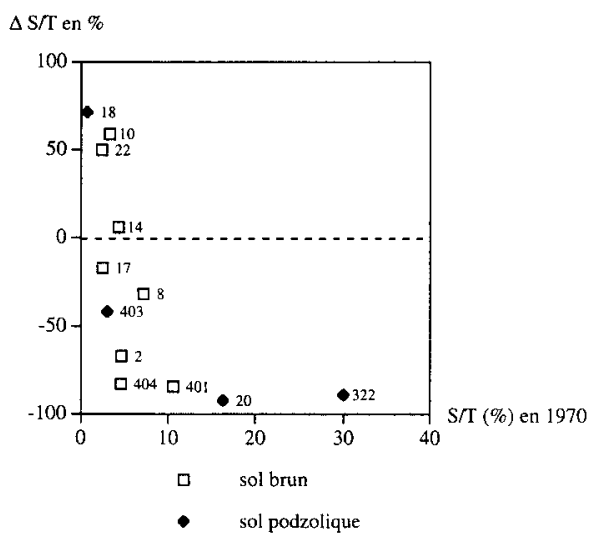

Fig 10. Variation du taux de saturation $(\Delta \mathrm{S} / \mathrm{T})$ entre 1970 et 1992 en fonction de sa valeur initiale dans le premier horizon minéral de la série Cartographie.

\section{DISCUSSION ET CONCLUSION}

L'évolution des horizons $A_{1}$ est essentiellement marquée par une baisse du taux de saturation. Si la perte de cations semble en être la principale cause, une augmentation non négligeable de la CEC s'observe dans un grand nombre de profils. Cette variation, liée à celle de la teneur en carbone organique, est particulièrement sensible dans les sols podzolisés de la série des Hospices de Nancy. Cette importante différence du taux de carbone pourrait provenir des défoliations subies par la sapinière dans les années 1980. À la suite de cet apport supplémentaire de litière, le stock de carbone a pu s'élever anormalement dans ce milieu où la minéralisation est lente. Cependant, l'amplitude de l'augmentation $\left(2,4 \%\right.$, soit $540 \mathrm{~kg} \cdot \mathrm{ha}^{-1} \cdot \mathrm{an}^{-1}$ supplémentaire de carbone dans les horizons $A_{1}$ ) ne peut pas être entièrement expliquée par ce seul phénomène. Il faut donc suspecter des problèmes de profondeur de rééchantillonnage. En effet, dans ces sols les horizons $\mathrm{H}$ se développent en relative continuité avec les horizons $A_{1}$. La teneur en matière organique varie très rapidement avec la profondeur ; en conséquence, une faible erreur sur l'épaisseur prélevée peut entrainer une grande variation sur le taux de carbone.

Ce biais d'échantillonnage est sans doute moins sensible dans la série Cartographie où les humus sont le plus souvent de type Mull ; en revanche, la gestion des peuplements a aussi des répercussions sur le statut carboné. Ainsi dans la station 18 , le maintien d'une régénération surabondante est probablement à l'origine de la forte accumulation de carbone. À l'inverse, une baisse du taux de carbone, par exemple dans la station 322, peut très bien provenir d'un passage en éclaircie qui a momentanément favorisé la minéralisation de la matière organique.

L'existence de ces fluctuations incite à la prudence dans l'affirmation d'une réelle dégradation. Néanmoins, dans la série Cartographie les variations de teneurs en calcium et magnésium sont significativement différentes dans les horizons $A_{1}$. De plus, la variabilité spatiale mesurée en 1992 dans ces horizons organominéraux est relativement faible, excepté dans une placette pour le calcium et dans deux autres pour le magnésium. Ces résultats rejoignent ceux d'une étude sur l'évolution des propriétés des sols des hêtraies du nord-est entre 1970 et 1991 (Thimonier, 1994). Sur un souséchantillon en milieu acide, cet auteur relève des pertes significatives en calcium et en potassium et une désaturation du complexe absorbant.

Il semble vraisemblable que, dans le contexte du massif vosgien, une aggravation sensible de l'état de désaturation du complexe absorbant s'est produite et cela sur une courte période. En prenant comme hypothèse une densité apparente de 0,9 , les pertes moyennes en $\mathrm{Ca}^{2+}$ et $\mathrm{Mg}^{2+}$ dans les horizons organominéraux peuvent être évaluées respectivement à 2,5 et $0,5 \mathrm{~kg} \cdot \mathrm{ha}^{-1} \cdot \mathrm{an}^{-1}$. Ces flux sont du même ordre de grandeur que ceux obtenus par des modélisations de bilan (Dambrine et al, 1995). 
Deux processus interviennent dans la désaturation observée : l'entraînement des cations hors de l'écosystème par drainage et l'immobilisation dans la biomasse. L'intensité et l'importance respective de ces deux flux varient selon la nature de l'écosystème considéré. L'âge du peuplement est l'un des facteurs principaux de cette variation. À Aubure (massif vosgien), l'immobilisation en calcium est estimée à $9,9 \mathrm{~kg} \cdot \mathrm{ha}^{-1} \cdot \mathrm{an}^{-1}$ et les pertes par drainage à $2,9 \mathrm{~kg} \cdot \mathrm{ha}^{-1} \cdot \mathrm{an}^{-1}$ sous une pessière de 30 ans, alors que sous une pessière de 85 ans le drainage devient plus important $\left(9,9 \mathrm{~kg} \cdot \mathrm{ha}^{-1} \cdot \mathrm{an}^{-1}\right)$ que l'immobilisation $\left(4,1 \mathrm{~kg} \cdot \mathrm{ha}^{-1} \cdot \mathrm{an}^{-1}\right)(\mathrm{Le}$ Goaster et al, 1991). Dans ce même site, les flux de magnésium se partagent par moitié entre le drainage $\left(1,4 \mathrm{~kg} \cdot \mathrm{ha}^{-1} \cdot \mathrm{an}^{-1}\right)$ et l'immobilisation (1,2 kg.ha-1 $\left.\mathrm{an}^{-1}\right)$ dans le jeune peuplement. Dans le plus âgé, en revanche, la part du drainage est bien plus élevée $\left(2,1 \mathrm{~kg} . \mathrm{ha}^{-1} \cdot \mathrm{an}^{-1}\right)$ que celle de l'immobilisation $\left(0,4 \mathrm{~kg} \cdot \mathrm{ha}^{-1} \cdot \mathrm{an}^{-1}\right)$. Dans une jeune pineraie du sud-est des États-Unis, Binkley et al (1989) n'attribuent que $10 \%$ des pertes totales en bases échangeables au drainage. Dans trois peuplements de douglas du Beaujolais, l'immobilisation en calcium varie entre 5,9 et $7,5 \mathrm{~kg} \cdot \mathrm{ha}^{-1} . \mathrm{an}^{-1}$ selon l'âge, mais d'importantes quantités de cet élément ( 12 kg.ha- $a^{-1} \cdot \mathrm{an}^{-1}$ ) sont relevées dans les eaux gravitaires profondes (Marques, 1996). Il s'agit là d'un écosystème relativement riche qui subit une évolution de la matière organique à la suite d'une substitution d'espèces. Sous une pessière de 50 ans, à Monthermé (massif des Ardennes), dans une région soumise à d'importants apports exogènes, les flux en calcium sont estimés à $14 \mathrm{~kg} \cdot \mathrm{ha}^{-1} \cdot \mathrm{an}^{-1}$ pour le drainage et à $6,8 \mathrm{~kg} \cdot \mathrm{ha}^{-1} \cdot \mathrm{an}^{-1}$ pour l'immobilisation. Ceux en magnésium le sont respectivement à 3,6 et 1,1 kg.ha- ${ }^{-1} \cdot \mathrm{n}^{-1}$ (Nys et al, 1983).

L’immobilisation d'éléments minéraux est d'autant moins importante que le peuplement est plus âgé. L'évolution des pertes par drainage au cours de la révolution forestière est beaucoup plus variable d'après les exemples cités. Dans la présente étude tous les peuplements étaient adultes dès 1970 , avec des âges moyens entre 80 et 120 ans. L'immobilisation dans la biomasse devrait donc être stabilisée. Toutefois, les études dendrochronologiques récentes sur le massif vosgien montrent que les productivités moyennes du sapin (Becker, 1987) et du hêtre (Picard, 1995) augmentent à long terme, et cela en liaison avec une évolution globale des conditions environnementales (climat, apports atmosphériques) (Becker et al, 1994). Quelles en sont les répercussions réelles sur le cycle des éléments ? Dans les sols pauvres, l'augmentation du prélèvement peut constituer à elle seule un phénomène important de désaturation, ou encore traduire un dysfonctionnement général de l'écosystème. Quoi qu'il en soit, qu'elle résulte d'exportations directes faisant suite à des apports acides d'origine exogène ou qu'elle soit due à un stockage à long terme dans la biomasse, l'acidification des sols étudiés, même si elle n'est pas totalement démontrée, est hautement probable.

L'interprétation des variations des stocks de carbone et d'azote est plus délicate. Quelles sont les parts respectives de l'erreur de l'échantillonnage, de la minéralisation ou des apports exogènes? Seule une tendance à l'accumulation d'azote peut être retenue, ce qui est compatible avec les apports atmosphériques observés sur le massif vosgien (Dambrine et al, 1993).

Les résultats exposés montrent que la baisse du calcium et du magnésium échangeables des sols acides étudiés n a pas entraîné une modification du pH eau. Cela signifie que ces sols ont encore une forte capacité à neutraliser les acides issus des apports atmosphériques ou générés par voie interne (cycle de l'azote). Les études réalisées sur un site voisin (col du Bonhomme) établissent que les solutions de sols sont cependant acides et que leur neutralisation définitive ne s'opère que dans la roche altérée sous-jacente (Mohamed Ahmed, 1992). 
Ces sols, développés sur substrat gréseux ou granitique, déjà fortement désaturés auraient subi une accentuation de leur appauvrissement au cours des deux dernières décennies. L'ensemble des données obtenues tend à démontrer cette hypothèse mais l'échantillonnage restreint et la variabilité spatiale limitent parfois la validité définitive des résultats. Corrélativement, ils auraient atteint des niveaux critiques pour la nutrition en particulier en magnésium, avec une teneur inférieure à $0,15 \mathrm{meq} / 100 \mathrm{~g}$ dans l'horizon $A_{1}$ (Landmann et al, 1995). Il s'agit là d'un phénomène préoccupant pour le caractère durable de l'aménagement de ces écosystèmes.

\section{REMERCIEMENTS}

Cette étude a été financée par la direction de l'Espace rural et de la Forêt du ministère de l'Agriculture. Nous remercions MM M Gury et D Merlet du Centre de pédologie biologique de Vandœuvre (CNRS) pour leur aide dans la recherche des sites.

\section{RÉFÉRENCES}

Becker M (1987) Bilan de santé actuel et rétrospectif du sapin (Abies alba Mill) dans les Vosges. Étude écologique ef dendrochronologique. Ann Sci For 44 , 379-402

Becker M, Bert GD. Bouchon J, Picard JF, Ulrich E, (1994) Tendances à long terme observées dans la croissance de divers feuillus et résineux du nord-est de la France depuis le milieu du XIX ${ }^{\mathrm{C}}$ siecle. Rey For Fr 46, 4

Binkley D. Valentine D. Wells C, Valentine U (1989) An cmpirical analysis of the factors contributing to 20-year decrease in soil $\mathrm{pH}$ in an old-field plantation ol loblolly pine. Biogeochemistry 8, 39-54

Bonneau M (1978) Les Basses-Vosges. In : Notice de la carte pédologique de la france an $1 / 100000$. $46-74$

Bonneau M (1989) Que sait-on maintenant des causes du « dépérissement " des forêts? Rev For Fr t1, 5

Chichery M (1970) Enquête sur les liaisons entre le milieu et la production de la sapinière sur granite du Valtin. Diplôme d'études approfondies. École nationale supérieure d’agronomie, Nancy, $40 \mathrm{p}$

Dambrine E. Bonneau M, Nourisson G ( 1993) Apport de pollution et de nutriments par l"atmosphère aux peuplements forestiers vosgiens : intensité. varia- tions spatiales et historiques et conséquences sur la nutrition des forêts. Ann Sci Rés Bios Vosges du Nord-3 (1993-1994), 7-24

Dambrine E. Sverdrup H. Warfvinge P (1995) Atmospheric deposition, forest management and soil nutrient availability: a modelling exercise. In : Forest Decline and Atmospheric Deposition Effects in the French Mountains. (G Landmann, M Bonneau eds). Springer-Verlag, Berlin, Heildelberg, New York, 259-269

Johnson DW, Cresser MS, Nilsson SL, Turner J, Ulrich B. Binkley D. Cole DW (1991) Soil changes in forest coosytems: evidence for and probable causes. Proc Royal Soc Edinburgh 97B, 81-116

Landmann G (1989) Évolution sur 20 ans de l'alimentation minérale d'un peuplement adulte de sapin pectiné et du complexe absorbant dans un site d'altitude du massil vosgien. Journées de travail Deforpa, Nancy-Paris, France, févr-mars 1989, 4. 10.3.110.3 .6

Landmann G, Bonneau M. Bouhot-Delduc L, Fromard F. Chéret V, Dagnac J, Souchier B (1995) Crown damage in Norway spruce and silver fir: relation to nutritional status and soil chemical characteristics in the French mountains. In : Forest Decline and Atmospheric Deposition Effects in the French Molmtains (G Landmann, M Bonncau, eds). Springer-Verlalg, Berlin, Heidelherg, New York, $41-81$

Le Goaster S, Dambrine F, Ranger J (1991) Croissance et nutrition d'un peuplement d'épicéa sur sol pauve. I. Évolution de la biomalsice et dynamique d'incorporation d'ćléments minéraux. Acta Oecol 12. $771-779$

Marques R (1996) Dynamique du fonctionnement minéral d'unc plantation de douglas (Pseudotsuga men-iesii (Mirb) Franco) dans les monts du Beaujolais (France). Thèse de l'École nalionale du génic rural, des eaux ct des lorêts, $240 \mathrm{p}$

Mohamed Amhed D (1992) Rôle du facteur édaphique dalls le fonctionnement biogéochimique et l'étal de santé de deux pessičres vosgiennes. Eflet d'un amendement calcimagnćsien. Thèse de l'université de Nancy-1

Nys C, Ranger D, Ranger J (1983) Étude comparative de deux écosystemes léuillus el résineux des Ardennes primaires. 1. Minéralomasse et cycle biologique d une pessière de 50 ans. Ann Sci for 40. $41-66$.

Picard JF (1995) Evolution de la croissance radiale du hêtre (Fagles silinatica L.) dans les Vosges. Premiers résultats sur le versant lorrain. Anm Sci For 52, 11 21

Tamm CO. Hallbäcken L (1988) Changes in soil acidity in two forest areas with different acid deposition: 1920s to 1980s. Ambio 17,56-61

Thimonier $A$ (1994) Changements de la végétation et des sols en forêl tempéréc européenne au cours de la période 197()-1990) : rôle possible des apports atmosphériques. Thèse de l'université d'Orsay. $178 \mathrm{p}$ 